\title{
Caracterización para la vinculación con la sociedad: caso de estudio Parroquia El Quinche
}

\author{
Fecha de recepción : 11-12-2020 • Fecha de aceptación: 23-02-2021 • Fecha de publicación: 10-05-2021
}

\author{
Carlos Eduardo Durán Chávez' \\ Universidad Metropolitana del Ecuador (UMET) \\ cduran@umet.edu.ec \\ https://orcid.org/0000-0002-9857-2220 \\ Marily Rafaela Fuentes Aguila² \\ Universidad Metropolitana del Ecuador (UMET) \\ mfuentes@umet.edu.ec \\ https://orcid.org/0000-0003-4242-8593 \\ Pamela Anahí Sandoval Yuqui ${ }^{3}$ \\ Universidad Metropolitana del Ecuador (UMET) \\ pamela.sandoval@est.umet.edu.ec \\ https://orcid.org/0000-0003-4064-9421
}

\section{Resumen}

El presente estudio tuvo como propósito evaluar la importancia que tienen los proyectos de investigación y vinculación con la sociedad en el Ecuador, como parte de las funciones sustantivas de la educación superior. En este sentido, se analizan las acciones realizadas a propósito de dichos proyectos, por parte de los estudiantes y profesores de la carrera de Derecho de la Universidad Metropolitana del Ecuador (UMET), enfocándose específicamente en la Parroquia rural El Quinche. Es una investigación de tipo jurídica, analítica y descriptiva, en la cual se aplicaron encuestas a los habitantes de la parroquia, para de esta manera poder obtener los resultados de los diferentes problemas que existen en este sector. En el análisis de las encuestas se muestra que una de las principales dificultades que afectan a la parroquia es la inseguridad, es por esto que se proponen algunas temáticas a tratar con el objetivo de orientar en la solución de los problemas observados en la zona. 
Palabras clave: vinculación con la sociedad, proyecto de investigación, caracterización, parroquia El Quinche.

\begin{abstract}
The purpose of this study was to evaluate the importance of research projects and outreach to society in Ecuador, as part of the substantive functions of higher education. In this sense, it analyzes the actions carried out by students and professors of the Law program of the Metropolitan University of Ecuador (UMET), focusing specifically on the rural parish of El Quinche. It is a legal, analytical and descriptive research, in which surveys were applied to the inhabitants of the parish, in order to obtain the results of the different problems that exist in this sector. The analysis of the surveys shows that one of the main difficulties affecting the parish is insecurity, which is why some issues are proposed to be addressed in order to guide the solution of the problems observed in the area.
\end{abstract}

Keywords: link with society, research project, characterization, El Quinche parish. 


\section{Introducción}

La vinculación con la sociedad es una de las tres funciones sustantivas de la educación superior, en conjunto con la docencia y la investigación, con el fin de contribuir a la solución de las necesidades y problemáticas del entorno desde el ámbito académico e investigativo, tal como lo prescriben el artículo 4 del Reglamento de Régimen Académico (Consejo de Educación Superior, 2019) y el artículo 117 de la Ley Orgánica de la Educación Superior (Asamblea Nacional, 2010b).

Esta se desarrolla mediante un conjunto de proyectos e iniciativas de interés público que son planificadas, ejecutadas, monitoreadas y evaluadas de manera sistemática por las Instituciones de Educación Superior (IES). La carrera de Derecho de la Universidad Metropolitana del Ecuador interviene con sus proyectos, de manera decisiva, en las comunidades de Voz Andes, Colinas del Norte y en la Parroquia de El Quinche, todas ubicadas en la provincia de Pichincha, en el Ecuador.

El Reglamento de Régimen Académico, plantea la necesidad y prescribe la obligación de las IES de poseer un modelo de vinculación con la sociedad en el cual se integren las tres funciones sustantivas mencionadas anteriormente. Con estos componentes las instituciones educativas y la propia vinculación se constituye en eje integrador de las funciones de la universidad.

En la Constitución de la República del Ecuador (Asamblea Nacional, 2008) se detalla en el artículo 350 la finalidad del sistema de educación superior, con la cual el Estado tiene como finalidad que se forme al estudiante académicamente y profesionalmente con una visión científica y humanista, preparando así profesionales aptos para enfrentarse al futuro. Desde el propio texto constitucional, se prevé la formación integral del alumno, no solamente en el ámbito académico, sino en valores humanos, culturales, éticos, solidarios, entre otros que permitan que su profesión sea útil a la sociedad y a su desarrollo económico, político y cultural.

La Ley Orgánica de Educación Superior (Asamblea Nacional, 2010) exige la materialización de estos procesos como requisitos para obtener los títulos universitarios correspondientes. En los artículos 13 y 87 se consagra la necesidad de garantizar la calidad de estos procesos.

Mientras que en el artículo 13, relativo a las funciones del Sistema de Educación Superior, se expresa que se debe garantizar el derecho a la educación superior mediante la docencia, la investigación y su vinculación con la sociedad, y asegurar crecientes niveles de calidad, excelencia académica y pertinencia. La integración de estos componentes permite el egreso de profesionales capaces de comprender con madurez la importancia de los saberes para la colectividad y el bien común.

A su vez, en el artículo 87 se establece como requisito previo a la obtención del título, que todos los estudiantes de educación superior deberán acreditar servicios a la comunidad por medio de prácticas o pasantías pre-profesionales en los campos de su especialidad. Tal exigencia viabiliza disminuir las tendencias individualistas de la muchas veces están impregnados los estudiantes de las diversas carreras, y otro que resulta importante mencionar es el artículo 88 (Asamblea Nacional, 2010), en este se precisa que, para cumplir con la obligatoriedad de los servicios a la comunidad, se propenderá a beneficiar a sectores rurales y marginados de la población, si 
la naturaleza de la carrera lo permite, o a prestar servicios en centros de atención gratuita. La atención que se realiza por la carrera de Derecho, en virtud de los proyectos de investigación y vinculación a las comunidades de Colinas del Norte, El Quinche y Voz Andes, da cumplimiento a los objetivos planteados por la Asamblea Nacional como órgano de poder del Estado ecuatoriano.

La vinculación con la sociedad se puede realizar mediante: servicios comunitarios, prestación de servicios especializados, consultorías, educación continua, entre otros, que facilitan la comunicación y la interacción de la Universidad Metropolitana del Ecuador con su entorno. Existen múltiples muestras de cómo los estudiantes y profesores visitan los sitios más recónditos en las comunidades, o acerca de la forma de vida de los indígenas y otros pobladores, de las encuestas y entrevistas realizadas a los habitantes y a los gobiernos autónomos, así como del apoyo de los gobiernos en la solución de los problemas planteados.

En esta investigación, se hace referencia a la Parroquia El Quinche, una de las rurales abordadas en atención a las actividades planificadas, tanto en el proyecto de investigación, como en el proyecto de vinculación con la sociedad. Las funciones sustantivas constituyen una labor misional por parte de la UMET, que posibilita ampliar el saber universitario y de esta manera sitúa a la universidad al servicio de la comunidad.

Cabe mencionar que los docentes y estudiantes del caso de estudio tienen asignadas tareas que emergen tanto de los proyectos de vinculación y el de investigación, que se han trasladado desde la caracterización y diagnóstico de las comunidades y parroquias hasta el planteo de soluciones a los problemas sociales identificados. De esta manera se promueve conjuntamente con la comunidad, el intercambio de conocimientos para generar un impacto positivo en el desarrollo sostenible del Ecuador.

\subsection{Los proyectos de investigación y sus fines}

La investigación es una de las funciones sustantivas de la educación superior, según el artículo 4 del Reglamento de Régimen Académico, este proceso se define como:

“(...) una labor creativa, sistemática y sistémica fundamentada en debates epistemológicos y necesidades del entorno, que potencia los conocimientos y saberes científicos, ancestrales e interculturales (...)" (Consejo de Educación Superior, 2019).

Partiendo del concepto anterior, se puede definir a los proyectos de investigación como procedimientos sistemáticos y reflexivos, diseñados con el fin de contribuir en un conocimiento generalizable, por otro lado, la Academia Nacional de Ciencias detalla que el objetivo de la investigación es "(...) extender el conocimiento humano acerca del mundo físico, biológico o social, más allá de lo ya conocido" (The Office of Search Integrity, 2019).

La investigación en las IES constituye un proceso diferente a otras maneras de hallar o detectar el conocimiento, por ejemplo, la lectura de un libro, visita a museos, mirar reportajes, etc. Debido a que utiliza un proceso llamado método científico, mediante la aplicación del mismo se obtiene 
información fidedigna y relevante que permite descubrir, explicar y generalizar los distintos fenómenos que se producen en la sociedad.

El método científico implica, en alguna de sus variantes, la observación del entorno que rodea y la formulación de hipótesis acerca de las relaciones que existen en el mismo. Cuando de manera mesurada se analiza una problemática, se concibe una posible respuesta anticipada al problema, la cual sirve como guía de trabajo para la investigación que se realice.

El trabajo que se presenta implicó la comprobación de la hipótesis y el examen de los resultados de las pruebas que tienen una estrecha vinculación con la hipótesis y el entorno. Con esta guía, el investigador puede interpretar la información que compila y llegar a conclusiones sólidas acerca de los resultados.

De acuerdo con el autor Ander-Egg (1995), los fines de los proyectos de investigación son descubrir o interpretar los hechos, fenómenos, relaciones y leyes de un determinado ámbito de la realidad, y; obtener conocimientos y solucionar problemas científicos, filosóficos o empíricotécnicos.

"Prevención del delito como estrategia del control social para la promoción de una cultura de paz" es el nombre que lleva el proyecto de investigación de la carrera de Derecho de la UMET. Dentro de sus objetivos se encuentra la caracterización y diagnóstico de las comunidades, entre ellas, la de la Parroquia rural El Quinche, con el fin de identificar los principales problemas que allí se producen y trazar acciones o estrategias que permitan disminuir los efectos negativos de la criminalidad.

Además de promover una cultura de paz y desarrollar las actividades relacionadas con la prevención del delito en dicha zona, aun cuando en las zonas no estaban diagnosticadas las dificultades más frecuentes que hoy se conocen. El proyecto de investigación ha fungido como una especie de investigación acción, en la medida en que los alumnos y grupos de trabajo han estado incidiendo en la educación, información y atención de las personas, desde las visitas primarias.

\subsection{Vinculación con la sociedad como parte de la investigación}

La tarea de las IES no se centra solo en realizar investigaciones o en ser un centro que guía y forma a futuros profesionales, sino que también estas deben poseer un componente mediante el cual se puedan relacionar las funciones mencionadas anteriormente, con las diversas realidades que posee nuestro entorno, es ahí donde aparece la vinculación con la sociedad, ya que a partir de los problemas detectados mediante las investigaciones previamente realizadas, los estudiantes, conjuntamente con sus docentes, pueden actuar de una manera clara, con el fin de proponer algunas acciones tendentes a solucionar los problemas que fueron detectados en la Parroquia, a través del proyecto de vinculación con la sociedad titulado "Promoción de la cultura de paz a través de la práctica de los métodos alternativos de solución de conflictos y de la consultoría jurídica gratuita que permita el acceso a la justicia", estrechamente relacionado con el proyecto de investigación de la carrera de Derecho antes mencionado. 
Por lo anterior, se afirma que las universidades, a través de sus proyectos de investigación y de vinculación con la sociedad, intervienen en los escenarios existentes en las comunidades, con el propósito de colaborar con dichas localidades rurales vulnerables en la solución de sus problemas, y de esta manera contribuir con la formación de los estudiantes -orientados por docentes- toda vez que estos, a través de sus prácticas investigativas y de vinculación con las comunidades, aportan y aplican el conocimiento adquirido durante sus años de estudio en la institución.

El filósofo latinoamericano Zea, establece que:

"La universidad está comprometida con la sociedad, es agente de cambio capaz de generar los conocimientos teóricos y prácticos que elevan la creación y recreación de sus individuos, necesarios para la solución de problemas a través de proyectos que estimulen la interacción recíproca universidad-comunidad, desarrollando la vida nacional en todos sus aspectos." (Zea, 1981).

Las instituciones de educación superior poseen la responsabilidad de generar conocimientos mediante la investigación, por tanto, la educación debe ser de calidad para de esta manera formar a profesionales responsables que puedan enfrentarse a los distintos retos que les depara el futuro, es por esto que la calidad se refleja en la exploración y en la producción de conocimiento que se proporciona al estudiante, no solo desde el punto de vista teórico, sino también práctico, con la puesta en acción de lo aprendido en las aulas.

Se entiende que las IES deben proponer orientaciones y respuestas a las necesidades sociales a través de una educación pertinente de procesos académicos dinámicos y comprometidos con el desarrollo humano, estableciendo vínculos con diversas organizaciones para proporcionar la atención debida a las poblaciones más vulnerables socio-económicamente, como es el caso de la Parroquia rural El Quinche.

Conforme a lo anterior, la vinculación con la sociedad se articula con la función sustantiva de docencia y la investigación para la formación integral de los estudiantes, quienes complementan la teoría con la práctica en los procesos de enseñanza-aprendizaje. La necesidad de que todos estos procesos se enlacen de manera efectiva es responsabilidad de todos los docentes y miembros de los proyectos.

La vinculación se enlaza con la investigación debido a que posibilita la identificación de necesidades y la formulación de preguntas que alimentan los proyectos de investigación, además, propicia el uso social del conocimiento científico y los saberes. La investigación académica y científica genera resultados que pueden ser utilizados en propuestas de vinculación con la sociedad, que beneficien la calidad de vida y el desarrollo social.

Por tal motivo, esta conlleva, en la mayoría de las IES, la realización de acciones de diversos géneros, caracterizadas por ubicarse fuera de las actividades académicas formales de las instituciones, aun cuando algunas de ellas constituyan un apoyo significativo a la docencia o a la investigación, $y$, por otra parte, por estar orientadas tanto a la comunidad interna de las instituciones, como a las que están fuera de ellas. 
Esta función sustantiva al interior de las Instituciones de Educación Superior, constituye según Fresán (2004), un proceso estratégico tanto en su capacidad de articular la docencia con la investigación y la preservación y difusión de sus saberes científicos, como en la formación integral de los estudiantes y de los demás miembros de la comunidad universitaria en un ambiente en el que la interlocución constituye la base de un proyecto orientado a la formación de individuos reflexivos y comprometidos con una sociedad menos injusta.

Desde el punto de vista normativo, la Constitución dispone en el artículo 350 que:

"El sistema de educación superior tiene como finalidad la formación académica y profesional con visión científica y humanista; la investigación científica y tecnológica; la innovación, promoción, desarrollo y difusión de los saberes y las culturas; la construcción de soluciones para los problemas del país, en relación con los objetivos del régimen de Desarrollo" (Asamblea Nacional Constituyente, 2008).

Es importante mencionar el artículo 107 de la LOES, que se refiere al principio de pertinencia, el cual consiste en que la educación superior responda a las expectativas y necesidades del desarrollo humano de la colectividad, local y regional, en manos de las organizaciones sociales locales, y de igual manera, en la planificación nacional, y al régimen del desarrollo humano general, la prospección de desarrollo científico, humanístico y tecnológico mundial, y la diversidad cultural. Las instituciones deben articular su oferta docente, de investigación y actividades vinculadas con la colectividad, a la demanda académica, a las necesidades de desarrollo local, regional y nacional.

Por su parte, el artículo 50 del Reglamento del Régimen Académico, dispone que:

"La vinculación con la sociedad hace referencia a la planificación, ejecución y difusión de actividades que garantizan la participación efectiva en la sociedad y responsabilidad social de las instituciones del Sistema de Educación Superior (...)" (Consejo de Educación Superior, 2019).

Así mismo, en el Reglamento de Régimen Académico, se declaran entre sus objetivos: articular y fortalecer la investigación; la formación académica y profesional; y la vinculación con la sociedad, en un marco de calidad, innovación y sostenibilidad que propenda al mejoramiento continuo (Consejo de Educación Superior, 2019, art. 3).

En consecuencia, de lo indicado, las actividades previstas en el proyecto de vinculación del estudio se relacionan estrechamente con las acciones planificadas en el proyecto de investigación de la carrera, constituyéndose ambos procesos sustantivos en fundamento necesario de las acciones que se realizan en las comunidades, en coordinación con organizaciones comunitarias, empresas e instituciones públicas y privadas. La participación de los estudiantes y docentes de la UMET, en prácticas de vinculación con la sociedad, proyectos comunitarios, educación continua y asesorías, servicios, etc. corresponde a los aprendizajes de las competencias específicas de cada carrera. 


\subsection{La Parroquia El Quinche: caracterización}

Según el Gobierno Parroquial El Quinche (2019), la palabra Quinche tiene origen en la lengua Maya "Cakchiquel", cuyo significado se compone de dos vocablos, Quin que significa Sol y Chi que significa Monte. Por tanto, apoyados en los aportes etimológicos de Manuel Moreno Mora, Quinche significa Monte del Sol.

Esta se encuentra ubicada a unas dos horas de Quito, y se puede llegar por dos diferentes rutas: Pifo o Guayllabamba (pasando por el zoológico de Quito). La ruta por Pifo es más corta y demora una hora y media. El Quinche forma parte del Distrito Metropolitano de Quito, ubicada en la zona noreste de la Provincia de Pichincha, en el Valle de Tumbaco y respecto al Cantón, se encuentra en la Zona Oriente del Cantón. Su ubicación según la latitud es: Latitud: S $0^{\circ} 10^{\prime}$ / S $0^{\circ} 0^{\prime}$ y Longitud: W 78 $30^{\prime} / \mathrm{W} 78^{\circ} 15^{\prime}$. Sus límites son por el Norte con la Parroquia Ascazubí, por el Sur con la Parroquia Checa; por el Este con la Parroquia Cangahua y la Reserva Ecológica CayambeCoca y por el Oeste con la Parroquia Guayllabamba.

En la planificación territorial de El Quinche, realizada por Global Consultora (2015), se han tomado en cuenta cuatro sectores, que se detallan a continuación:

SECTOR A. Denominado montañoso, se caracteriza por su baja densidad poblacional, falta de infraestructura en general, por mantener un territorio con relieves que conforman el páramo alcanzando las máximas alturas del territorio, por su paisaje natural con vocación en la silvicultura y conservación ambiental, y por su constante apego al área periurbana de donde se abastece principalmente de todos los servicios.

SECTOR B. Denominada zona periurbana, su denominación está configurada por su semejanza al habitual transitar del espacio urbano; geográficamente está situado de manera adyacente a la ciudad principal, se caracteriza por ser un territorio en constante transformación, con expectativas, frágil y susceptible a nuevas intervenciones, con un mayor asentamiento humano y con consideraciones en el desarrollo de infraestructuras y de servicios.

SECTOR C. Se denomina zona rural por su aspecto de paisaje agrario y por la influencia de sus principales actividades agropecuarias y agroindustriales. Alberga la mayor parte de las estaciones de producción, por lo cual se diferencia de las zonas de montañas y periurbana.

SECTOR D. Es la zona rural de baja densidad poblacional con vocación agrícola. Se diferencia del Sector $\mathrm{C}$ por su carencia de estructura productiva a nivel agropecuario y agroindustrial, alberga asentamientos humanos dispersos con infraestructura casi completa, y con una determinación a ser parte del territorio que cambia y se transforma.

Esta parroquia es una de las más pobladas del Cantón, cuenta con un total de 16056 habitantes según el Instituto Nacional de Estadística y Censos (INEC) en el último censo del año 2010, los sábados y domingos recibe de 5.000 a 10.000 personas, y los meses de mayo y noviembre visitan más de 400.000 personas debido al atractivo turístico que es La Virgen de El Quinche. Actualmente el principal atractivo que presenta la ciudad es su Iglesia, consagrada a la Virgen 
del mismo nombre, santuario de adoración a la virgen de El Quinche, la cual fue traída desde Oyacachi en el año 1604. Cada año hay romerías a pie desde Quito para visitar a la Virgen, conocida por ser muy milagrosa.

La mayoría de personas que residen en este sector son mestizos, pero al igual que todas las poblaciones existen asentamientos, de afroecuatorianos, indígenas y algunas personas que migran hacia esta población.

La Parroquia El Quinche se rige por el Código Orgánico de Organización Territorial, Autonomía y Descentralización (COOTAD), está bajo el régimen de un Gobierno Autónomo Descentralizado (GAD), por lo tanto, están en la obligación de promocionar el desarrollo y garantizar el buen vivir por medio de la gestión de sus competencias. El ejercicio de los gobiernos autónomos descentralizados se realiza a través de tres funciones integradas:

a) De legislación, normatividad y fiscalización;

b) De ejecución y administración; y,

c) De participación ciudadana y control social (Asamblea Nacional, 2010, art. 29).

El viernes 2 de junio del año 2017, la Universidad Metropolitana del Ecuador, conjuntamente con el Gobierno Autónomo Descentralizado de la Parroquia de El Quinche, suscribió un Convenio Interinstitucional a través del cual se realizan procesos de vinculación con la población. Esta se basa en un proceso de inter aprendizaje, donde los académicos brindan el conocimiento de la ciencia y la técnica; mientras que la comunidad aporta con sus saberes, los que, unificados en la práctica, proyectan actividades conjuntas para conocer y transformar la realidad. La educación superior se encuentra llamada a contribuir en el desarrollo local y nacional de manera permanente, a través del trabajo comunitario o vinculación con la sociedad. En este sentido, un grupo de docentes, conscientes de la responsabilidad social que tiene la universidad con la sociedad, crearon una campaña para capacitar y fomentar la "Cultura de Paz" en los sectores más apartados de la administración de justicia ordinaria, como lo son las parroquias rurales.

El objetivo principal de esta campaña es promover esta cultura a través de la utilización de métodos alternativos en la resolución de conflictos, para lo cual un grupo de estudiantes universitarios realizaron una investigación de campo, mediante un acercamiento directo con la población de la referida parroquia, para determinar los principales problemas que atraviesan en la resolución de sus conflictos. Cuyos resultados fueron reflejados en un folleto informativo.

\section{Metodología}

La investigación se define como "un conjunto de procesos sistemáticos y empíricos que se aplican al estudio de un fenómeno o problema” (Hernández, Fernández y Baptista; 2014, p. 18).

Este estudio se desarrolló a través de una metodología mixta, debido a que reúne elementos teóricos y empíricos que contribuyen, por una parte, a aportar a la doctrina criminológica y por 
otra, a constatar algunos hechos y elementos prácticos que sustentan la teoría del control social específicamente en la comunidad estudiada.

De acuerdo con los autores Hernández, Fernández y Baptista (2014, p. 30) la investigación mixta "es un enfoque que implica combinar los métodos cuantitativo y cualitativo en un mismo estudio"

Mientras que Blasco y Pérez (2007, p. 25), refieren que "la investigación cualitativa estudia la realidad en su contexto natural y cómo sucede, sacando e interpretando fenómenos de acuerdo con las personas implicadas".

Por su parte, Hurtado y Toro (1998, p. 16), señalan que "la investigación cuantitativa tiene una concepción lineal que implica claridad entre los elementos que conforman el problema, que deben ser limitados y saber con exactitud donde inician, también se debe reconocer qué tipo de incidencia existe entre sus elementos"

Por tanto, la combinación de estos dos enfoques de investigación implica la complementariedad entre ambos, necesaria para abordar el fenómeno estudiado desde distintas perspectivas.

El enfoque mixto (cuali-cuantitativo) se constituye en una "vía para que el investigador pueda acercarse a su objeto de estudio y obtener el conocimiento requerido de la realidad social según sus necesidades y expectativas" (Del Canto y Silva, 2013, p. 26).

La investigación se llevó a cabo en correspondencia con las líneas de investigación que tributan al proyecto de investigación de la carrera de Derecho de la UMET en Quito, relacionado con la prevención del delito para la promoción de una cultura de paz, que abarcó tres zonas rurales fundamentales entre las cuales se encuentra la Parroquia El Quinche.

Durante el año 2017, los estudiantes aplicaron encuestas a las personas residentes de la comunidad rural. La referida encuesta tuvo como objetivo obtener información para desarrollar la caracterización de la comunidad objeto de la investigación y confeccionar el diagnóstico criminológico comunitario. De igual forma, fueron aplicadas entrevistas a informantes clave y a representantes de la comunidad.

Los resultados obtenidos sobre la situación delictiva en la comunidad, pueden generar un conjunto de elementos, directrices y referentes, para la identificación de la situación criminológica en otros ciudadanos o comunidades del territorio nacional.

\section{Resultados}

Luego de ser analizados los resultados de las entrevistas y encuestas aplicadas en la comunidad rural El Quinche, estos permitieron confirmar lo siguiente:

\subsection{Diagnóstico criminológico}


- La comunidad desconocía sobre de las posibles prácticas de vinculación con la sociedad realizadas por universidades cercanas a las comunidades en cuestión. Destaca el desconocimiento acerca de las posibles asesorías que pueden realizar las carreras de Derecho, mediante la asesoría y consultoría jurídica gratuita para los sectores de la población con menos capacidad económica.

- La población desconocía los conceptos relacionados con los servicios jurídicos y su alcance a los miembros de la comunidad que los pudiera necesitar (pensión alimenticia, régimen de visitas, tenencia y custodia de menores, delitos, entre otros).

- Existió consenso en la necesidad de consultoría para atender la violencia intrafamiliar y otros delitos.

- La mayoría desconocía acerca de la existencia de los métodos alternativos de solución de conflictos para acometer las necesidades de atención jurídica, por ejemplo, la mediación.

- De igual forma, se desconocía que las universidades que tienen carreras de Derecho, podían prestar el servicio de consultoría jurídica gratuita.

- El desconocimiento de los conceptos y servicios jurídicos conllevaba a su vez la desinformación sobre las autoridades a donde podían acudir para denunciar posibles hechos delictivos.

- Del resultado anterior se desprendió que la mayoría optaba por la solución de actos de violencia mediante la "violencia por mano propia".

- No existía una concepción sólida en cuanto al sistema de valores de la sociedad y los responsables (instituciones) de su desarrollo.

- Se reconocía la existencia de la inseguridad ciudadana ocasionada por la existencia de grupos informales y lugares inseguros.

- Insuficientes espacios y centros para la recreación y esparcimiento de las personas, así como de acciones por parte de las autoridades de los Gobiernos Autónomos Descentralizados (GAD's) u Organizaciones No Gubernamentales (ONG's) dirigidas a la recreación sana de los jóvenes con el fin de prevenir la comisión de delitos. Sin embargo, llamó la atención del grupo de investigación de la UMET, la existencia de muchos establecimientos en los cuales se expenden bebidas alcohólicas en la comunidad.

- La parroquia carecía de centros de capacitación para contribuir a la solución de conflictos sociales en la comunidad, de la misma manera no existían organizaciones en la comunidad que promovieran la coexistencia pacífica y el respeto mutuo.

- Existían opiniones encontradas en relación a la posible influencia positiva o negativa de los medios masivos de difusión en el desarrollo de actitudes violentas. 
- No existían acciones preventivas contra la adicción y consumo de alcohol y drogas, tampoco se apreciaron acciones para lograr el acercamiento sano entre grupos de jóvenes.

- La mayoría de las víctimas de violencia identificadas fueron los adultos mayores.

- Los conflictos que generaban la mayor violencia en la comunidad son los de tipo intrafamiliar, condiciones de vida (la insalubridad, carencias de alumbrado público, servicios de salud, etc.), violencia entre cónyuges (femicidio y homicidio).

- Se comprobó el desconocimiento por parte de la población en cuanto a qué es la prevención del delito y la labor de la policía en el control y prevención del mismo.

- Existen grupos informales de antisociales dedicados al expendio y consumo de drogas en diferentes lugares de la comunidad.

- Los encuestados consideraron necesario realizar acciones comunitarias para prevenir y controlar el delito entre las que señalan: mayor patrullaje policial, realización de charlas y capacitación en los temas de prevención y consecuencias del delito.

- Entre los delitos más comunes se destacan: el robo, asesinato, femicidio, consumo y tráfico de drogas, entre otros.

- Se considera la existencia de suficientes centros de educación y el acceso de la población a los mismos, aunque no se apreció la relación directa de los centros educativos con la prevención del delito.

Estos resultados muestran como uno de sus principales problemas es la inseguridad. Esta situación coloca a la zona en un estado de vulnerabilidad a pesar de la necesidad de que en la localidad exista un ambiente de paz y tranquilidad, de forma tal que se favorezca el flujo de personas que visitan asiduamente la iglesia del Quinche y sus alrededores.

La Universidad Metropolitana, consciente de su encargo social en el ámbito de la vinculación con la sociedad, se dispuso a la creación de un plan de acciones que estarían dirigidas a mejorar o aliviar el clima desfavorable que informan sus habitantes. Así, los grupos de trabajo emprendieron con actividades concretas que tuvieran una incidencia positiva en la población.

\subsection{Impacto de la universidad en la Parroquia El Quinche}

Con los resultados obtenidos de las encuestas, durante el primer trimestre de 2018, la UMET, consecuente con la misión y el compromiso de proponer algunas alternativas para acometer los problemas evidenciados en la parroquia, se realizó una planificación de actividades de capacitación que serían desarrolladas en el seno de la Parroquia El Quinche.

De acuerdo a la información obtenida de la investigación, se propusieron las siguientes temáticas que serían de gran ayuda para este sector: 
- Prevención del delito

- Alfabetización en la cultura de paz

- Métodos alternativos de solución de conflictos

- Consultoría jurídica gratuita

Para efectuar estas actividades de capacitación, resultó necesaria la interacción de las dos partes, es decir, por un lado, la participación de los habitantes de la parroquia y por otro lado, el aporte de los estudiantes y profesores de la UMET que están a cargo del proyecto de vinculación.

Con el desarrollo de las temáticas propuestas, se pretendió generar cambios en la colectividad de la parroquia, en relación con los tópicos que fueron abordados en las capacitaciones. Los moradores del sector se mostraron accesibles y apoyaron la ejecución de las actividades que desarrolla la universidad para el bien de toda la comunidad.

Con el objetivo de comunicar las necesidades de intervención en la comunidad, por parte de los estudiantes para colaborar en la prevención del delito, se realizó un taller en el que se comunicaron los resultados del diagnóstico. Participaron dirigentes comunitarios, pobladores, docentes y estudiantes de la carrera de Derecho.

La segunda acción estuvo dirigida a que los estudiantes proporcionaran información a la población de la parroquia acerca de los servicios que brida el centro de mediación de la universidad, para lo cual se elaboraron diferentes iniciativas y formas de información, ya fuese atendiendo directamente a los interesados en la propia comunidad y a través de la información, a viva voz, en reunión de la comunidad, ejemplificando los casos que pueden llevarse a la mesa de mediación y cómo pueden resolverse los conflictos más frecuentes que se presentan en la zona, como es el caso de fijación de pensiones alimenticias, cobros de deudas, entre otros. Se logró poner en contacto a los habitantes en caso de que necesiten de los servicios de asesoría y mediación que proporciona la UMET.

Para que los residentes de El Quinche conocieran los términos relacionados con los posibles servicios jurídicos a los que pueden acceder en caso de necesitarlos, los estudiantes prepararon y ofrecieron capacitación a la comunidad en relación con los conceptos jurídicos que fueron revelados en las encuestas como desconocidos. Se debatieron los temas de prevención, causas de la criminalidad, relación entre la educación y la prevención, la cultura, la recreación y el deporte como medio de prevención.

Se elaboraron materiales de divulgación para darle a conocer a los pobladores los horarios, fechas, datos de los responsables y hora de atención por los centros de mediación y consultorios jurídicos.

Finalmente, el plan de acciones trazó la tarea de aplicar encuestas nuevamente para medir el grado de satisfacción de los habitantes, con las medidas adoptadas. De tal modo, se cumple el 
objetivo de validar las actividades realizadas por los estudiantes y docentes en el proyecto.

Las actividades de los proyectos de vinculación e investigación de la carrera de Derecho en Quito aún no culminan en El Quinche. Tanto los docentes como alumnos que participan en la citada comunidad liderados por los docentes Eugenio Égüez Valdivieso, Osvaldo Brito Febles y Aura Díaz de Perales, se encuentran comprometidos con tareas muy concretas asociadas a la prevención y atención social a las necesidades de la zona. Los vínculos de solidaridad y compromiso entre la universidad y el Gobierno del Quinche imponen nuevos retos en favor de la solución a los problemas más urgentes en la comunidad, se atienden nuevos proyectos educativos, se trabaja en el mejoramiento de la recreación a los niños y adolescentes y se prevé la profundización del estudio de las causas que han provocado determinados delitos.

La entrega que representan los proyectos de vinculación e investigación en la comunidad constituye un ejemplo a seguir por los docentes en el ámbito universitario. El año 2020 se pudieron mostrar con total nitidez los resultados definitivos de un trabajo sostenido de la carrera de Derecho a partir de las investigaciones que se realizan en el orden criminológico sobre causas, condiciones del delito, perfil criminológico de las personas que delinquen, medios de control social y su perfeccionamiento en la zona caso de estudio.

\section{Conclusiones}

Según los resultados obtenidos, en la Parroquia EI Quinche existen varios problemas sociales que afectan el buen desarrollo de la comunidad, ya que situaciones como la inseguridad, violencia, entre otros, han sido factores que obstaculizan el buen vivir dentro de la parroquia. Conforme a lo anterior, los habitantes esperan que exista más participación y control de las autoridades pertinentes, en relación con la problemática observada.

Por otra parte, en cuanto a los proyectos de investigación y vinculación con la sociedad, los mismos se constituyen como instrumentos de gran importancia para las comunidades, tal como se ha presentado en esta investigación, toda vez que tanto estudiantes como docentes, proponen alternativas reales para la solución de la problemática estudiada, procurando así generar resultados e impactos significativos en las comunidades. De igual forma, los estudiantes de la carrera de Derecho de la UMET, practicantes en estos proyectos, desarrollan competencias investigativas y teórico-prácticas, conforme a lo cual se relacionan las tres funciones sustantivas principales (docencia, investigación y vinculación con la sociedad) permitiéndoles aplicar los conocimientos adquiridos aportándole a la sociedad.

Conforme a lo anterior, las Instituciones de Educación Superior, al implementar el aprendizaje experimental y vivencial, propician una gran herramienta de generación de conocimientos y favorecen el intercambio de saberes entre profesores, estudiantes y comunidades. 


\section{Referencias}

Ander-Egg, E. (1995). Técnicas de investigación social. Buenos Aires, Argentina: Editorial LUMEN.

Blasco, J. \& Pérez, J. (2007). Metodologías de investigación en educación física y deporte: Ampliando horizontes. Alicante, España. Editorial Club Universitario. Imprenta Gamma.

Del Canto, E \& Silva, A. (2013.) Metodología Cuantitativa: Abordaje desde la complementariedad en ciencias sociales. Revista de Ciencias Sociales 141: 25-34 / 2013 (III) ISSN: 0482-5276. https://doi.org/10.15517/ rcs.v0i141.12479

Ecuador. Asamblea Nacional (2010a). Código Orgánico de Organización Territorial, Autonomía y Descentralización. Registro Oficial 303, de fecha 19 de octubre de 2010. Última modificación: 23 de octubre de 2018. Quito: Asamblea Nacional.

Ecuador. Asamblea Nacional (2010b). Ley Orgánica de Educación Superior. Registro Oficial 298, de fecha 12 de octubre de 2010. Última modificación: 02 de agosto de 2018. Quito: Asamblea Nacional.

Ecuador. Asamblea Nacional Constituyente. (2008). Constitución de la República del Ecuador. Registro Oficial 449, de fecha 20 de octubre de 2008. Última modificación: 30 de abril de 2019. Quito: Asamblea Nacional Constituyente.

Ecuador. Consejo de Educación Superior. (2019). Reglamento de Régimen Académico. Registro Oficial 473, de fecha 23 de abril de 2019. Quito: Consejo de Educación Superior.

Ecuador. Gobierno Parroquial El Quinche (2019). Datos generales de la parroquia El Quinche. http://elquinche. gob.ec/

Fresán, M. (2004). La extensión universitaria y la Universidad Pública. Distrito Federal, México: Universidad Autónoma Metropolitana.

Hernández, R. Fernández, C \& Baptista, P. (2014). Metodología de la investigación. Sexta edición. México: McGraw-Hill

Hurtado, I \& Toro, J. (1998). Paradigmas y métodos de investigación en tiempos de cambio. Segunda edición. Valencia, Venezuela: Ediciones de la Universidad de Carabobo.

The Office of Search Integrity. (2019). ¿Qué es la Investigación? ORI. https://ori.hhs.gov/m\%C3\%B3dulo-1-introducci\%C3\%B3n-\%C2\%BFqu\%C3\%A9-es-investigaci\%C3\%B3n

Zea, L. (1981). Sentido de la Difusión Cultural Latinoamericana. Distrito Federal, México: Universidad Nacional Autónoma de México 
Copyright (c) 2021 Carlos Eduardo Durán Chávez, Marily Rafaela Fuentes Aguila y Pamela Anahí Sandoval Yuqui

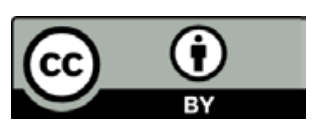

Este texto está protegido bajo una licencia internacional Creative Commons 4.0.

Usted es libre para Compartir-copiar y redistribuir el material en cualquier medio o formato

- y Adaptar el documento - remezclar, transformar y crear a partir del material-para cualquier propósito, incluso para fines comerciales, siempre que cumpla las condiciones de Atribución. Usted debe dar crédito a la obra original de manera adecuada, proporcionar un enlace a la licencia, e indicar si se han realizado cambios. Puede hacerlo en cualquier forma razonable, pero no de forma tal que sugiera que tiene el apoyo del licenciante o lo recibe por el uso que hace de la obra.

\section{$\underline{\text { Resumen de licencia - Texto completo de la licencia }}$}

